University of Nebraska - Lincoln

DigitalCommons@University of Nebraska - Lincoln

April 2002

\title{
Coercive fields in ferroelectrics: A case study in lithium niobate and lithium tantalate
}

\section{Sungwon Kim}

Pennsylvania State University, University Park

Venkatraman Gopalan

Pennsylvania State University, University Park, Pennsylvania, gopalan@matse.psu.edu

Alexei Gruverman

University of Nebraska-Lincoln, agruverman2@unl.edu

Follow this and additional works at: https://digitalcommons.unl.edu/physicsgruverman

Part of the Physics Commons

Kim, Sungwon; Gopalan, Venkatraman; and Gruverman, Alexei, "Coercive fields in ferroelectrics: A case study in lithium niobate and lithium tantalate" (2002). Alexei Gruverman Publications. 8.

https://digitalcommons.unl.edu/physicsgruverman/8

This Article is brought to you for free and open access by the Research Papers in Physics and Astronomy at DigitalCommons@University of Nebraska - Lincoln. It has been accepted for inclusion in Alexei Gruverman Publications by an authorized administrator of DigitalCommons@University of Nebraska - Lincoln. 


\title{
Coercive fields in ferroelectrics: A case study in lithium niobate and lithium tantalate
}

\author{
Sungwon Kim and Venkatraman Gopalan ${ }^{\mathrm{a})}$ \\ Materials Research Institute and Department of Materials Science and Engineering, Pennsylvania State \\ University, University Park, Pennsylvania 16802
}

\begin{abstract}
Alexei Gruverman
Department of Materials Science and Engineering, North Carolina State University, Raleigh, North Carolina 27695
\end{abstract}

(Received 26 November 2001; accepted for publication 25 February 2002)

\begin{abstract}
The experimentally measured coercive electric fields for domain reversal in ferroelectrics are typically many orders of magnitude lower than the estimates from phenomenological free-energy theory. This letter specifically investigates the influence of polarization gradients at pre-existing $180^{\circ}$ domain walls in ferroelectrics on coercive fields for domain wall motion. It is shown that the ratio of theoretical coercive field without and with a preexisting domain wall is directly proportional to the ratio $x_{o} / a$, where $a$ is the lattice parameter and $2 x_{o}$ is the polarization wall width. This factor is $7.5-45$ for a $20-120 \mathrm{~nm}$ wall width, the latter width determined here as the experimental upper limit for polarization wall width in lithium tantalate. (C) 2002 American Institute of Physics.
\end{abstract}

[DOI: $10.1063 / 1.1470247]$

Why are the experimentally measured coercive fields for domain reversal in ferroelectrics many orders of magnitude smaller than theoretical estimates? The question mirrors a similar one asked with regard to mechanical strength of materials, namely, why is, for example, the measured critical resolved shear stress in materials (e.g., $\sim 10^{10} \mathrm{~N} / \mathrm{m}^{2}$ in metals) many orders of magnitude lower than experimentally observed deformation shear stresses (e.g. $\sim 10^{6} \mathrm{~N} / \mathrm{m}^{2}$ in metals). ${ }^{1}$ This discrepancy in mechanical strength is explained by the presence of dislocations in materials. The discrepancy in ferroelectrics has primarily been discussed through the recognition that while the simple estimate of intrinsic coercive fields from the Landau-Ginzburg (LG) theory does not account for the presence of domain walls or other defects, real single domain crystals nonetheless possess defect sites where it is easier to nucleate domains. ${ }^{2,3}$ Once a domain nuclei exists, the Miller and Weinreich theory ${ }^{4}$ explains the effective lateral motion of pre-existing, atomically sharp domain walls, in terms of the probability of overcoming an energy barrier, $U$, to preferentially nucleate small wedge-shaped domains adjoining the wall. Though not uniquely defined, one can consider the maximum electric field, $E=d U / d P$ required to completely overcome the steepest climb out of the energy well, $U$ versus $P$ (polarization), as an estimate of the intrinsic coercive field. Domain wall pinning at physical defects can also contribute to the experimental coercive fields, which manifests as the threshold field for wall depinning. ${ }^{5}$

In this letter, we explore the theoretical estimate of coercive field from the LG theory in the presence of preexisting $180^{\circ}$ domain walls with finite wall widths. Specifically, we wish to show that a finite polarization gradient at the domain wall can lower the local intrinsic coercive fields adjacent to the wall, thereby facilitating local wall motion.

${ }^{a)}$ Electronic mail: vgopalan@psu.edu
The problem is treated generally for any ferroelectric with a second-order phase transition, and two possible antiparallel domain states. As specific examples, we consider ferroelectric lithium niobate $\left(\mathrm{LiNbO}_{3}\right)$ and lithium tantalate $\left(\mathrm{LiTaO}_{3}\right)$, which are technologically important, ${ }^{6,7}$ and typically contain pre-existing domain nuclei in nominally singlecrystal, single-domain crystals. ${ }^{8,9}$

Let us first calculate the theoretical coercive field for $180^{\circ}$ domain reversal in these materials. The GinzburgLandau-Devonshire free energy per unit volume, $G$, of a ferroelectric with a second order phase transition is given $b^{2}$

$$
G=-\frac{\alpha_{1}}{2} P^{2}+\frac{\alpha_{2}}{4} P^{4},
$$

where the order parameter is the polarization, $P$. One can calculate the effective $\alpha_{1}$ and $\alpha_{2}$ coefficients from the dielectric constant $\varepsilon_{33}$ and the homogeneous spontaneous polarization, $P_{s}$ as $\alpha_{1}=1 /\left(2 \varepsilon_{33}\right)$ and $\alpha_{2} \sim \alpha_{1} / P_{s}^{2}$. These values at room temperature are $\varepsilon_{33}=43.5 \varepsilon_{o}$ and $P_{s}=0.55 \mathrm{C} / \mathrm{m}^{2}$ for $\mathrm{LiTaO}_{3}, \varepsilon_{33}=30 \varepsilon_{o}, P_{s}=0.75 \mathrm{C} / \mathrm{m}^{2}$, for $\mathrm{LiNbO}_{3}$. The equation of state, $E=d G / d P$ defines the theoretical $P$ versus $E$ hysteresis loop. The coercive field $E=E_{c}$ is defined as the turning point $(d E / d P)_{E=E_{c}}=0$, which determines the polarization $P_{c}$ at the coercive field as $P_{c}= \pm \sqrt{\alpha_{1} /\left(3 \alpha_{2}\right)}$. Substituting this value back in the equation of state yields the coercive field $E_{c}$ as

$$
E_{c}= \pm \frac{2}{3 \sqrt{3}} \sqrt{\frac{\alpha_{1}^{3}}{\alpha_{2}}} \sim \pm 0.385 \alpha_{1} P_{s}
$$

Substituting for the values of $\alpha_{1}$ and $\alpha_{2}$ in Eq. (2), the intrinsic coercive fields are $E_{c} \sim 2750 \mathrm{kV} / \mathrm{cm}\left(\mathrm{LiTaO}_{3}\right)$ and $E_{c} \sim 5420 \mathrm{kV} / \mathrm{cm}\left(\mathrm{LiNbO}_{3}\right)$. In contrast, the coercive fields reported in literature for near-stoichiometric $\mathrm{LiTaO}_{3}$ is 


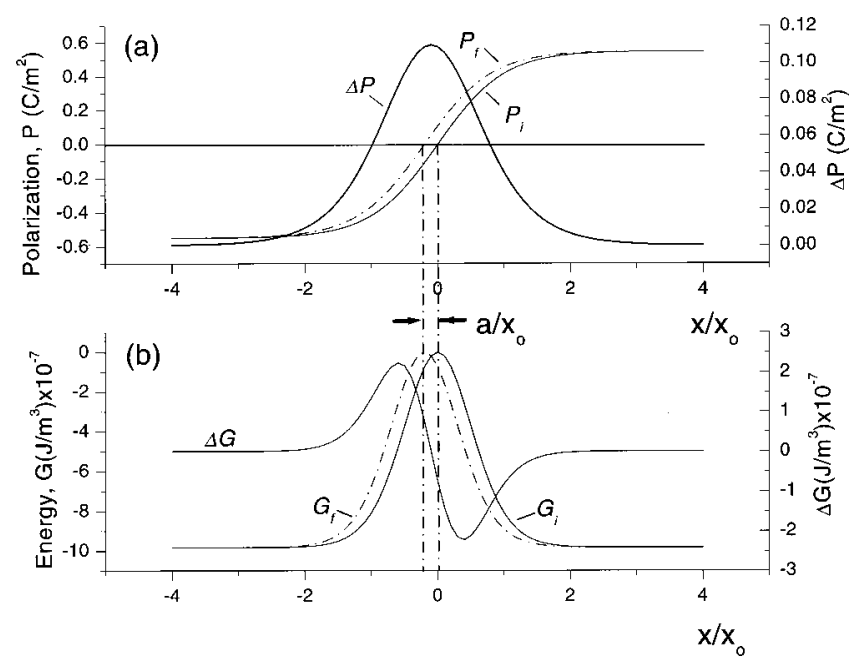

FIG. 1. (a) A plot of the polarization gradients [Eq. (3)], $P_{i}$ (initial), $P_{f}$ (final), and $\Delta P=P_{f^{-}} P_{i}$, of a $180^{\circ}$ domain wall in $\mathrm{LiTaO}_{3}$ after movement of the wall by an amount $a$ under the influence of an external field $\mathrm{E}$ in the $+x$ direction. (b) The corresponding polarization energies [Eq. (1)], $G_{i}$, and $G_{f}$, and $\Delta G=G_{f} G_{i}$ are also shown. The normalization term $x_{o}$ is the domain half width according to Eq. (3).

$\sim 17 \mathrm{kV} / \mathrm{cm},{ }^{10}$ for near-stoichiometric $\mathrm{LiNbO}_{3}$ is $\sim 40 \mathrm{kV} / \mathrm{cm},{ }^{11}$ and for congruent compositions of both materials is $\sim 210 \mathrm{kV} / \mathrm{cm}$. $^{10,11}$

In the following analysis, we now consider the presence of pre-existing $180^{\circ}$ domain walls with a finite wall width of polarization gradient, $P(x)$ defined $\mathrm{as}^{2}$

$$
P=P_{s} \tanh \left(x / x_{o}\right),
$$

where $P_{s}$ is the spontaneous polarization, $x$ is coordinate normal to the wall and $2 x_{o}$ is the characteristic wall width. This polarization, $P$ and the associated free energy, $G$ [from Eq. (1)] is plotted in Fig. 1(a) for $\mathrm{LiTaO}_{3}$. Now we ask the following question: What is the free energy change $\Delta G$ when such a domain wall moves by one lattice parameter, $\Delta x=a$, resulting in a change of polarization and energy at every location $x$ by amounts $\Delta P=P_{f}-P_{i}$ and $\Delta G=G_{f}-G_{i}$ ? (Subscripts $i$ and $f$ stand for initial and final states, respectively, as shown in Fig. 1). One can numerically calculate these quantities. On the other hand, if one assumed that $a / x_{o}$ is small, then, one can analytically evaluate $\Delta P$ $=(d P / d x) \Delta x$ from Eq. (3). The corresponding free energy change is calculated as $\Delta G=(\partial G / \partial P) \Delta P$, using Eq. (1) and $\Delta P$. For example, from Fig. 1, it is clear that for the wall to move in the $-x$ direction, there is a positive energy barrier, $\Delta G$ that the external field, $+E$ has to overcome, which is less than the original energy barrier, $G$ for a graded wall. The minimum external field required to overcome this barrier is therefore, the steepest slope of the energy well $\Delta G$ versus $P$, i.e., $E_{c}^{\prime}=(-d(\Delta G) / d P)_{\max }$. This occurs at the wall center $(x=0)$. The negative sign reflects the negative slope of $\Delta G$ versus $P$ at the wall center under a positive field $+E$ (see Fig. 1). The coercive field for domain motion can therefore be simplified at the wall center as

$$
E_{c}^{\prime} \sim P_{s} \alpha_{1} a / x_{o},
$$

where $K=P_{s} \alpha_{1} a$ was determined as $0.368 \mathrm{~V}$ for $\mathrm{LiTaO}_{3}$ and 0.726 volts for $\mathrm{LiNbO}_{3}$. Clearly, the coercive field contribution for this type of domain wall motion is inversely
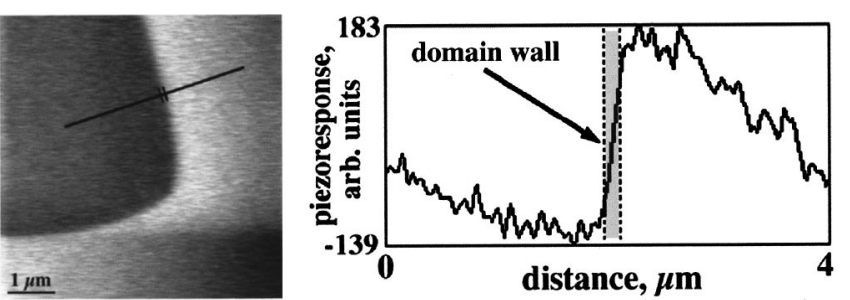

FIG. 2. First harmonic piezoresponse signal image (left-hand side) of one vertex of a triangular $180^{\circ}$ domain in a congruent $\mathrm{LiTaO}_{3}$ crystal using a scanning probe microscope. Polarization is normal to the image plane. The cross section profile of the piezoresponse signal across the domain wall (i.e., along the black line in the image) is shown on the right-hand side. The imaging voltage of $5 \mathrm{~V}, 10 \mathrm{kHz}$ was applied using a standard Au-coated $\mathrm{Si}_{3} \mathrm{~N}_{4}$ cantilever with a spring constant of $0.1 \mathrm{~N} / \mathrm{m}$ and a resonant frequency of $34 \mathrm{kHz}$. The probing tip with an apex curvature radius of about $20 \mathrm{~nm}$ was in mechanical contact with the sample surface during the measurements (repulsive force regime). The image scan rate was $0.5 \mathrm{~Hz}$.

proportional to the wall width, $2 x_{o}$. Out of curiosity, if we presently ignore other mechanisms, and calculate the values of the equivalent domain wall widths, $x_{o}$ required to account for experimentally measured coercive fields in nearstoichiometric lithium niobate ${ }^{11}$ and lithium tantalate, ${ }^{10}$ we arrive at the upper limits of domain wall widths of $x_{o}$ $\sim 216 \mathrm{~nm}$ for near-stoichiometric $\mathrm{LiTaO}_{3}$ and $x_{o} \sim 181 \mathrm{~nm}$ for near-stoichiometric $\mathrm{LiNbO}_{3}$. For congruent compositions, these widths would be $17.5 \mathrm{~nm}\left(\mathrm{LiTaO}_{3}\right)$ and $34.5 \mathrm{~nm}$ $\left(\mathrm{LiNbO}_{3}\right)$.

Are these polarization widths at all reasonable? On the one hand, first-principle calculations on $180^{\circ}$ domain walls suggest that they may be just a unit cell $(\sim 0.4 \mathrm{~nm})$ wide. $^{12}$ On the other hand, recent work on domain walls in $\mathrm{LiNbO}_{3}$ and $\mathrm{LiTaO}_{3}$ show unexpectedly wide regions of $\operatorname{strain}^{13}$ and optical birefringence (extending over micrometers) around individual domain walls, which are strongly correlated with the presence of nonstoichiometry in the crystal. ${ }^{5}$ Further, we have recently conducted in situ x-ray synchrotron experiments of individual domain wall strains under external fields in lithium niobate single crystals, which clearly show that extended domain wall strains develop over 10-50 $\mu \mathrm{m}$ around the wall at fields an order of magnitude below experimental coercive fields. One naturally enquires if the corresponding polarization gradient at the domain walls might be broadened as well in these materials due to nonstoichiometry or in the presence of an external field. A piezoelectric scanning probe microscopy image of domain walls in congruent $\mathrm{LiTaO}_{3}$ is shown in Fig. 2. The principle of domain imaging in the piezoresponse mode is described in detail elsewhere. ${ }^{14}$ Figure 2 also shows a cross section profile of the piezoresponse signal across the domain wall (i.e., along the black line in the image). Due to possible artifacts related to sample leveling, we refer not just to the distance between maximum and minimum values of the piezoresponse signal (which is $\sim 400 \mathrm{~nm}$ wide) but also to the actual image of the wall, which was found to be about $120 \mathrm{~nm}$. The best resolution that could be expected can be as small as the radius of the tip-sample contact area, i.e., of the order of several nanometers. However, the observed widths should be treated as upper limits until a detailed modeling of the image is performed.

In addition to wall broadening, other factors can contrib- 
ute to coercive fields. Taking some these into account, we can therefore write the net experimentally observed coercive fields as,

$$
E_{c}=E_{c}^{\prime}-E_{\mathrm{sc}}+E_{\text {defect }},
$$

where $E_{\mathrm{sc}}$ is the space-charge field, and $E_{\text {defect }}$ is the bulk dipolar defect field. Surface polarization relaxation ${ }^{15}$ can give rise to a polarization gradient and hence a space-charge layer near the crystal surface as recently observed in $\mathrm{LiNbO}_{3} \cdot{ }^{16,17}$ Even if the space-charge density (charge/area) in this layer is $\sigma \sim 10^{-2}-10^{-3} P_{s}$, the space-charge field, $E_{\mathrm{sc}} \sim \sigma / \varepsilon$ would be of the same order of magnitude as the experimental coercive fields in lithium niobate and tantalate, and will aid in domain wall motion. The dipolar defects, which stabilize domains, such as defect complexes of Ta or $\mathrm{Nb}$ antisites and lithium vacancies proposed in recent literature, ${ }^{10}$ will give rise to equivalent defect fields, $E_{\text {defect }}{ }^{18}$ which tend to increase the coercive fields. As noted in Refs. 10 and 18, the defect field $E_{\text {defect }}$ is not an existing electric field in the material. Rather, it is a formal equivalent to the energetic difference between the two domain states $+P_{s}$ and $-P_{s}$, one stabilized by the dipolar defects by an amount of energy $-E_{\text {defect }} \cdot \mathrm{P}_{\mathrm{s}}$ and another raised in energy by the same amount. Therefore, a very likely scenario for near-stoichiometric crystals, $\left(E_{\text {defects }} \sim 0\right)$ is that the experimentally measured coercive field, $E_{c}$, may be equal to the net sum of the first two terms in Eq. (5) (and perhaps further contributions arising from other types of defects). Since the stoichiometric composition of $\mathrm{LiTaO}_{3}$ has a coercive field of $\leqslant 17 \mathrm{kV} / \mathrm{cm}$, the defect field in the congruent composition would be $\geqslant 210-17=193 \mathrm{kV} / \mathrm{cm}$, which clearly dominates the experimentally observed coercive fields $(210 \mathrm{kV} / \mathrm{cm})$ in congruent compositions.

The important conclusion of this letter is that, compared with theoretical estimates, the local coercive field in the presence of a polarization gradient at a $180^{\circ}$ domain wall is reduced by a factor of $\sim 0.385 x_{o} / a$, where $a$ is the lattice parameter and $2 x_{o}$ is the wall width. More generally, one could expect that any significant polarization fluctuation in a ferroelectric crystal, not just limited to a domain wall, would potentially reduce the coercive field for domain reversal in that region. The precise experimental determination of polarization wall width (particularly in the presence of small amounts of nonstoichiometry and under external fields) is central to resolving the issue of theoretical coercive fields in ferroelectric crystals.

This work was supported by the National Science Foundation Grant Nos. 9984691 and 9988685.

${ }^{1}$ Physical Metallurgy Principles, edited by R. E. Reed-Hill, 3rd ed. (PWSKent, Boston, MA, 1991).

${ }^{2}$ Principles and Applications of Ferroelectrics and Related Materials, edited by M. E. Lines and A. M. Glass (Clarendon, Oxford, 1977).

${ }^{3}$ S. Ducharme, V. M. Fridkin, A. V. Bune, S. P. Palto, L. M. Blinov, N. N. Petukhova, and S. G. Yudin, Phys. Rev. Lett. 84, 175 (2000); Also see comments by A. M. Bratkovsky and A. P. Levanyuk, ibid. 87, 019701 (2000); S. Ducharme and V. M. Fridkin, ibid. 87, 019702 (2000).

${ }^{4}$ R. C. Miller and G. Weinreich, Phys. Rev. 117, 1460 (1960).

${ }^{5}$ T. J. Yang, U. Mohideen, V. Gopalan, and P. Swart, Phys. Rev. Lett. 82, 4106 (1999)

${ }^{6}$ R. G. Hunsperger, Integrated Optics: Theory and Technology, 4th ed. (Springer, New York, 1995).

${ }^{7}$ R. L. Byer, Nonlinear Opt. 6, 549 (1997).

${ }^{8}$ V. Gopalan and T. E. Mitchell, J. Appl. Phys. 83, 941 (1998).

${ }^{9}$ H. Cerva, P. Pongratz, and P. Stalicky, Philos. Mag. A 54, 199 (1986).

${ }^{10}$ S. Kim and V. Gopalan, J. Appl. Phys. 90, 2949 (2001).

${ }^{11}$ V. Gopalan, T. E. Mitchell, Y. Furukawa, and K. Kitamura, Appl. Phys. Lett. 72, 1981 (1998).

${ }^{12}$ J. Padilla, W. Zhong, and D. Vanderbilt, Phys. Rev. B 53, R5969 (1996).

${ }^{13}$ S. Kim, B. Steiner, and V. Gopalan, Appl. Phys. Lett. 77, 2051 (2000).

${ }^{14}$ A. Gruverman, O. Auciello, and H. Tokumoto, Annu. Rev. Mater. Sci. 28, 101 (1998); A. Gruverman, O. Auciello, and H. Tokumoto, J. Vac. Sci. Technol. B 14, 602 (1996).

${ }^{15}$ J. Padilla and D. Vanderbilt, Phys. Rev. B 56, 1625 (1997).

${ }^{16}$ R. Williams, J. Phys. Chem. Solids 26, 399 (1965).

${ }^{17}$ Y. Cho, S. Kazuta, and H. Ito, Appl. Phys. Lett. 79, 2955 (2001).

${ }^{18}$ G. Arlt and H. Neumann, Ferroelectrics 87, 109 (1998). 